\title{
$c$-SECTIONS, SOLVABILITY AND LARGE SUBGROUPS OF FINITE GROUPS
}

\author{
BARBARA BAUMEISTER ${ }^{\bowtie}$ and GIL KAPLAN
}

(Received 22 October 2011)

\begin{abstract}
$c$-Sections of maximal subgroups in a finite group and their relation to solvability have been extensively researched in recent years. A fundamental result due to Wang ['C-normality of groups and its properties', J. Algebra 180 (1998), 954-965] is that a finite group is solvable if and only if the $c$-sections of all its maximal subgroups are trivial. In this paper we prove that if for each maximal subgroup of a finite group $G$, the corresponding $c$-section order is smaller than the index of the maximal subgroup, then each composition factor of $G$ is either cyclic or isomorphic to the O'Nan sporadic group (the converse does not hold). Furthermore, by a certain 'refining' of the latter theorem we obtain an equivalent condition for solvability. Finally, we provide an existence result for large subgroups in the sense of Lev ['On large subgroups of finite groups' J. Algebra 152 (1992), 434-438].
\end{abstract}

2010 Mathematics subject classification: primary 20E34; secondary 20E28, 20D05, 20D10.

Keywords and phrases: $c$-section, solvability, maximal subgroup, large subgroup.

\section{Introduction}

All groups in this paper are finite. Most of our notation is standard. For $A \leq G$ we denote the class of all the subgroups conjugate to $A$ in $G$ by $\operatorname{Con}_{G}(A)$. If $A \leq G$ and $|A| \geq|G|^{1 / 2}$ then $A$ is called a large subgroup of $G$.

Let $M$ be a maximal subgroup of a group $G$ and $K / L$ be a chief factor of $G$ such that $L \leq M$ while $K \not \leq M$. Following Shirong and Wang in [5], we call the group $(M \cap K) / L$ a $c$-section of $M$. It was proved [5, Theorem 1.1] that for a fixed maximal subgroup $M$ of $G$ all the $c$-sections of $M$ are isomorphic. We denote the abstract group isomorphic to a $c$-section (and so to all $c$-sections) of $M$ by $\operatorname{Sec}(M)$.

In [6] it was proved (although not using this terminology) that a group is solvable if and only if the $c$-sections of all its maximal subgroups are trivial. Further solvability conditions were proved in [5]. In particular, a group is solvable if and only if the $c$-sections of all its maximal subgroups are 2-closed [5, Theorem 2.1], and if and only if the $c$-sections of all its maximal subgroups are nilpotent [5, Theorem 2.2]. The case when all the $c$-sections are supersolvable was discussed in [4].

(c) 2012 Australian Mathematical Publishing Association Inc. 0004-9727/2012 \$16.00 
In this paper we study further the notion of $c$-sections and its connection to solvability. In particular, for a maximal subgroup $M$ we consider the relation between the order of the $c$-section $|\operatorname{Sec}(M)|$ and the index $|G: M|$. By the above, if $G$ is solvable then obviously $|\operatorname{Sec}(M)|<|G: M|$ for each maximal subgroup $M$ of $G$. It turns out that the converse is not true.

ExAmple 1.1. Let $T=O^{\prime} N a n$, the O'Nan simple sporadic group, and let $G=\operatorname{Aut}(T)=$ $T: 2$. We show that $|\operatorname{Sec}(M)|<|G: M|$ for all maximal subgroups $M$ of $G$. If $M=T$ then $|\operatorname{Sec}(M)|=1<|G: M|=2$. Let $M$ be maximal in $G, M \neq T$. Since $T / 1$ is a chief factor of $G$ and $M \nsupseteq T, M>1$, we have $S:=\operatorname{Sec}(M)=M \cap T$. By $G=M T$ it follows that for each $g \in G$ there exists $t \in T$ such that $S^{g}=S^{t}$. Thus $\operatorname{Con}_{T}(S)=\operatorname{Con}_{G}(S)$, and so $\operatorname{Con}_{T}\left(N_{T}(S)\right)=\operatorname{Con}_{G}\left(N_{T}(S)\right)$. Assume now that $|\operatorname{Sec}(M)| \geq|G: M|$. Then $|S| \geq|G: M|$, implying that $|S| \geq|T: S|$ and $|S| \geq|T|^{1 / 2}$, that is, $S$ is a large subgroup of $T$. By checking the list of maximal subgroups of $T=O^{\prime} N a n$ in [2], we deduce that $S$ is contained in a maximal subgroup of $T$ isomorphic to $L_{3}(7): 2$. Considering the maximal subgroups of $L_{3}(7): 2$, it follows that the only possibilities are $S \cong L_{3}(7): 2$ and $S \cong L_{3}(7)$, and in any case $N_{T}(S) \cong L_{3}(7): 2$. By the information in [2] we deduce that $\operatorname{Con}_{T}\left(N_{T}(S)\right) \neq \operatorname{Con}_{G}\left(N_{T}(S)\right)$, contradicting our previous observation. Thus $|\operatorname{Sec}(M)|<|G: M|$ for all maximal subgroups $M$ of $G$.

The involvement of $O^{\prime} N a n$ in Example 1.1 is not a coincidence. We have the following result.

THEOREM 1.2. Let $G$ be a group such that $|\operatorname{Sec}(M)|<|G: M|$ for all maximal subgroups $M$ of $G$. Then every composition factor of $G$ is either cyclic or isomorphic to O'Nan.

The converse of Theorem 1.2 is not true. Indeed for $G=O$ 'Nan there exists a large maximal subgroup $M$, so that $|\operatorname{Sec}(M)|=|M| \geq|G: M|$. Actually, it was proved in [3] that each simple nonabelian group has a proper large subgroup (and hence a large maximal subgroup). A key step in proving Theorem 1.2 is the following proposition.

Proposition 1.3. Let $G$ be a simple nonabelian group. Then the following are equivalent:

(1) $G$ has a proper large subgroup $H$ such that $\operatorname{Con}_{G}(H)=\operatorname{Con}_{\mathrm{Aut}(G)}(H)$;

(2) $G \supsetneqq O ’ N a n$.

By a certain 'refinement' of Theorem 1.2, we get an equivalent condition for solvability in Theorem 1.4 below. Throughout this paper, we write

$$
\beta:=\log (175560) / \log (2624832) \simeq 0.817 .
$$

(This number is connected to the largest proper subgroup $H$ of $G=O$ ' $N a$ an satisfying $\operatorname{Con}_{G}(H)=\operatorname{Con}_{\operatorname{Aut}(G)}(H)$.)

Theorem 1.4. Let $G$ be a group. Then $G$ is solvable if and only if $|\operatorname{Sec}(M)|<|G: M|^{\beta}$ for all maximal subgroups $M$ of $G$. 
We show in Proposition 2.8 that the (nonsolvable) group $G=\operatorname{Aut}\left(O^{\prime} N a n\right)$ satisfies $|\operatorname{Sec}(M)| \leq|G: M|^{\beta}$ (with equality in some cases) for all maximal subgroups $M$ of $G$. Thus $\beta$ cannot be replaced by a larger constant in Theorem 1.4.

Next, we include the following result, which, unlike the other results in this paper, is 'classification-free'.

THEOREM 1.5. Let $G$ be a group. Then the following are equivalent.

(1) $|\operatorname{Sec}(M)|<|G: M|$ for all maximal subgroups $M$ of $G$.

(2) For each nonabelian chief factor $K / L$ of $G$, and for each $L<B<K$ such that $B / L$ is large in $K / L, \operatorname{Con}_{K}(B) \neq \operatorname{Con}_{G}(B)$.

Let $G$ be a group satisfying the conditions of Theorem 1.5. We note that, by our Theorem 1.2, it follows that each noncyclic composition factor of $G$ (if exists) is isomorphic to $O^{\prime} N a n$.

The main result of [3] is that each group of composite order has a proper large subgroup. By applying Proposition 1.3 we prove the following theorem.

THeOREM 1.6. Let $G$ be a group such that $|G|$ is divisible by at least two primes. Assume that $G$ does not have composition factors isomorphic to O'Nan. Then $G$ has a proper large subgroup $H$ such that $\operatorname{Con}_{G}(H)=\operatorname{Con}_{\operatorname{Aut}(G)}(H)$.

The restriction on the composition factors of $G$ in Theorem 1.6 cannot be removed. This is clearly demonstrated by considering $G=O^{\prime}$ 'Nan. Furthermore, the statement of this theorem does not hold in general for $p$-groups (where $p$ is a prime), as can be shown by the example of any elementary abelian $p$-group.

The proof of Proposition 1.3 is given in Section 2. The proofs of Theorems $1.2,1.4,1.5$ and 1.6 are given in Section 3.

\section{Proof of Proposition 1.3}

Notice first that in Example 1.1 we showed that if $T=O^{\prime} N a n$ and $S$ is a proper large subgroup of $T$, then $\operatorname{Con}_{T}(S) \neq \operatorname{Con}_{\text {Aut }(T)}(S)$. Thus the implication $(1) \Rightarrow(2)$ of Proposition 1.3 is proved. It remains to prove that each simple nonabelian group $G$, except $O$ 'Nan, has a proper large subgroup $H$ satisfying $\operatorname{Con}_{G}(H)=\operatorname{Con}_{\operatorname{Aut}(G)}(H)$. We prove this separately for the sporadic simple groups, the simple groups of Lie type and the alternating groups; see Proposition 2.1, Corollary 2.4 and Proposition 2.5, respectively.

Proposition 2.1. Let $G$ be a sporadic simple group which is not isomorphic to O'Nan. Then $G$ has a proper large subgroup $H$ such that $\operatorname{Con}_{G}(H)=\operatorname{Con}_{\operatorname{Aut}(G)}(H)$.

Proof. As mentioned above, it was proved in [3] that each simple nonabelian group $G$ has a large maximal subgroup. When $\operatorname{Out}(G)=1$ this large subgroup $H$ certainly satisfies our extra condition. In Table 1 we give for each sporadic group $G$ 
TABle 1. Large subgroups $H$ such that $\operatorname{Con}_{G}(H)=\operatorname{Con}_{\text {Aut }(G)}(H)$.

\begin{tabular}{llrr}
\hline$G$ & $H$ & $|H|$ & $|G: H|$ \\
\hline$M_{12}$ & $L_{2}(11)$ & 660 & 144 \\
$M_{22}$ & $L_{3}(4)$ & 20160 & 22 \\
$S u z$ & $G_{2}(4)$ & 251596800 & 1782 \\
$H S$ & $M_{22}$ & 443520 & 100 \\
$M^{C} L$ & $U_{4}(3)$ & 3265920 & 275 \\
$H e$ & $S_{4}(4): 2$ & 1958400 & 2058 \\
$H N$ & $A_{12}$ & 239500800 & 1140000 \\
$J_{2}$ & $U_{3}(3)$ & 6048 & 100 \\
$J_{3}$ & $L_{2}(16): 2$ & 8160 & 6156 \\
$F i_{22}$ & $2 \cdot U_{6}(2)$ & 18393661440 & 3510 \\
$F i_{24}^{\prime}$ & $F i_{23}$ & 4089470473293004800 & 306936 \\
\hline
\end{tabular}

with $\operatorname{Out}(G)>1$, except $O^{\prime} N a n$, a corresponding large maximal subgroup $H$ such that $\operatorname{Con}_{G}(H)=\operatorname{Con}_{\operatorname{Aut}(G)}(H)$. This information is based on [2], and completes the proof.

Recall that a Borel subgroup $B$ of a group of Lie type $G$ in characteristic $p$ is the normaliser of a Sylow $p$-subgroup of $G$. Since the Sylow $p$-subgroups of $G$ are conjugate in $G$, it follows that $\operatorname{Con}_{G}(B)=\operatorname{Con}_{\text {Aut }(G)}(B)$. The following proposition states that in most cases $B$ is large in $G$. We have not found a reference for this property, which may be of independent interest. For brevity of notation, we say that the twisted group of Lie type ${ }^{\sigma} \mathcal{L}_{l}\left(q^{\sigma}\right)$ is defined over the field $\operatorname{GF}(q)$.

Proposition 2.2. Let $G$ be a simple group of Lie type ${ }^{\sigma} \mathcal{L}_{l}\left(q^{\sigma}\right)$ of rank l defined over the field with q elements, where $q>2$. Then a Borel subgroup $B$ of $G$ is a large subgroup of $G$.

Proof. We deal separately with the cases when $G$ is twisted or not.

Case 1. Let $G$ be a nontwisted group of Lie type. Then according to [1, 9.4.10],

$$
|G|=\frac{1}{d} q^{N}\left(q^{d_{1}}-1\right) \cdots\left(q^{d_{l}}-1\right), \quad|B|=\frac{1}{d} q^{N}(q-1)^{l}
$$

and

$$
|G: B|=\left(q^{d_{1}}-1\right) \cdots\left(q^{d_{l}}-1\right) /(q-1)^{l},
$$

where $d$ is as in [1,9.4.10], $N=\left|\Phi^{+}\right|$is the number of positive roots of the root system related to $G$ and $d_{1}+\cdots+d_{l}=N+l[1,9.3 .4]$.

By assumption $q \geq 3$. Assume that $l=1$. Then $q \geq 4$ is even, $N=1$ and $d_{1}=$ $N+l=2$. Hence

$$
|G: B|=\left(q^{2}-1\right) /(q-1)=q+1 \quad \text { and } \quad|B|=q(q-1) /(q-1,2) .
$$

As $q(q-1) \geq 3 q$ and $3 q>2(q+1)$, the assertion follows. 
Now let $l \geq 2$. If $l=2$ and $q=3$, then either $d=1$ and $G \cong L_{3}(3)$ or $G_{2}(3)$, or $d=2$ and $G \cong \mathrm{PS}_{4}(3)$. In the first case $|B|=2^{2} \cdot 3^{3}$ or $2^{2} \cdot 3^{6}$ and $|G: B|=2^{2} \cdot 13$ or $2^{4} \cdot 7 \cdot 13$, respectively. Thus $B$ is a large subgroup of $G$. If $G \cong \operatorname{PS} p_{4}(3)$ then $|B|=2 \cdot 3^{4}=162$ and $|G: B|=2^{5} \cdot 5=160$ and the assertion holds again.

From now on we assume that $l \geq 3$ if $q=3$ and $l \geq 2$ otherwise. We aim to show that

$$
\left(q^{d_{1}}-1\right) \cdots\left(q^{d_{l}}-1\right)<\frac{1}{d} q^{N}(q-1)^{2 l}
$$

We have $\left(q^{d_{1}}-1\right) \cdots\left(q^{d_{l}}-1\right)<q^{\sum_{i=1}^{l} d_{i}}=q^{N+l}$ and claim that $(q-1)^{2 l-1}>q^{l}$, which then yields the assertion. First let $q=3$. Then $l \geq 3,\left(\frac{4}{3}\right)^{l}>2$ and so $2^{2 l-1}>3^{l}$ as required. Now suppose that $q \geq 4$. Then $(q-1)^{2 l}>\left(q^{2}-2 q\right)^{l}=q^{l}(q-2)^{l}$. Thus it remains to show that $(q-2)^{l} \geq q-1$. This holds, as $(q-2)^{l} \geq(q-2)^{2}=q^{2}-4 q+4$ and $q^{2} \geq 5(q-1)$.

Case 2. Now let $G$ be a twisted group of Lie type. We choose to retain the notation of [1]. So $G$ is isomorphic to one of the following groups:

$$
{ }^{2} A_{l}\left(q^{2}\right),{ }^{2} B_{2}\left(q^{2}\right),{ }^{2} D_{l}\left(q^{2}\right),{ }^{3} D_{4}\left(q^{3}\right),{ }^{2} E_{6}\left(q^{2}\right),{ }^{2} F_{4}\left(q^{2}\right),{ }^{2} G_{2}\left(q^{2}\right),
$$

where $q^{2}=2^{2 m+1}$ (respectively, $q^{2}=3^{2 m+1}$ ) if $\mathcal{L}$ is of type $B_{2}$ or $F_{4}$ (respectively, of type $G_{2}$ ).

Let $B$ be a Borel subgroup of $T$. Then by [1, 14.1.2],

$$
|B|=\frac{1}{d} q^{N}\left(q-\eta_{1}\right)\left(q-\eta_{2}\right) \cdots\left(q-\eta_{l}\right),
$$

where $N$ is the number of positive roots in the root system related to $\mathcal{L}_{l}(q), d$ will be indicated in each case and $\eta_{1}, \ldots, \eta_{l}$ are the eigenvalues of the isometry $\tau$ of the vector space spanned by the roots which is related to the symmetry of the diagram for $\mathcal{L}_{l}(q)$. By [1, 14.3.2] we know $|G|$ and can calculate the index $|G: B|$ in all cases. We now discuss all the possibilities.

Let $G \cong{ }^{2} A_{l}\left(q^{2}\right)$ be a unitary group. We distinguish between the cases $l$ even and $l$ odd.

If $l$ is even, then

$$
d=(q+1, l+1), \quad N=\frac{l(l+1)}{2}, \quad \eta_{1}=\cdots=\eta_{l / 2}=1, \quad \eta_{l / 2+1}=\cdots=\eta_{l}=-1 .
$$

So

$$
|B|=\frac{1}{d} q^{l(l+1) / 2}(q-1)^{l / 2}(q+1)^{l / 2}
$$

and

$$
|G: B|=\prod_{i=1}^{l} \frac{q^{i+1}-(-1)^{i+1}}{(q-1)^{l / 2}(q+1)^{l / 2}} .
$$


Notice that $\left(q^{m}-1\right)\left(q^{m+1}+1\right)<q^{m+m+1}$. Thus

$$
|G: B|<\frac{q^{2+3+\cdots+(l+1)}}{(q-1)^{l / 2}(q+1)^{l / 2}}=\frac{q^{(l(l+1) / 2)+l}}{(q-1)^{l / 2}(q+1)^{l / 2}} .
$$

So it is enough to show that

$$
q^{l} \leq \frac{1}{d}(q-1)^{l}(q+1)^{l}, \quad \text { that is, } q \leq \frac{1}{d^{1 / l}}(q-1)(q+1) .
$$

Since the 'worst' case is $d=q+1$, it suffices to show that $q \leq(q-1)(q+1)^{1-1 / l}$. Since in fact $q \leq(q-1)(q+1)^{1 / 2}$ for $q>2$, we are done.

If $l \geq 3$ is odd, then

$$
d=(q+1, l+1), \quad N=\frac{l(l+1)}{2}, \quad \eta_{1}=\cdots=\eta_{(l+1) / 2}=1
$$

and

$$
\eta_{((l+1) / 2)+1}=\cdots=\eta_{l}=-1 .
$$

So

$$
|B|=\frac{1}{d} q^{l(l+1) / 2}(q-1)^{(l+1) / 2}(q+1)^{(l-1) / 2}
$$

and

$$
|G: B|=\prod_{i=1}^{l} \frac{q^{i+1}-(-1)^{i+1}}{(q-1)^{(l+1) / 2}(q+1)^{(l-1) / 2}} .
$$

Similarly to the previous case, we obtain $|G: B|<q^{(l(l+1) / 2)+l} /(q-1)^{(l+1) / 2}(q+1)^{(l-1) / 2}$. Thus it is enough to show that $q^{l} \leq(1 / d)(q-1)^{l+1}(q+1)^{l-1}$. Again we take the worst case $d=q+1$, so it suffices to show that $q^{l} \leq(q-1)^{l+1}(q+1)^{l-2}$, that is, $q^{l} \leq\left(q^{2}-1\right)^{l-2}(q-1)^{3}$. As $q<q^{2}-1$, it suffices to show that $q^{3}<\left(q^{2}-1\right)(q-1)^{3}$. Since the latter holds for every $q>2$, this case is concluded as well.

Let $G \cong{ }^{2} B_{2}\left(q^{2}\right)$ be a Suzuki group. Then $d=1, N=4, \eta_{1}=1$ and $\eta_{2}=-1$. Thus

$$
|B|=q^{4}\left(q^{2}-1\right), \quad|G: B|=q^{4}+1
$$

and the assertion holds for every $q$.

Let $G \cong{ }^{2} D_{l}\left(q^{2}\right)$ be an orthogonal group of minus type. Then $d=\left(4, q^{l}+1\right), N=$ $l(l-1), \eta_{1}=\cdots=\eta_{l-1}=1$ and $\eta_{l}=-1$. Thus

$$
|B|=\frac{1}{d} q^{l(l-1)}(q-1)^{l-1}(q+1)
$$

and

$$
|G: B|=\frac{\left(q^{l}+1\right) \prod_{i=1}^{l-1}\left(q^{2 i}-1\right)}{(q-1)^{l-1}(q+1)} .
$$


Then

$$
|G: B|<q^{l-1} 2^{l-1} \prod_{i=1}^{l-1} q^{2 i-1}=2^{l-1} \prod_{i=1}^{l-1} q^{2 i}=2^{l-1} q^{l(l-1)} \leq q^{l(l-1)}(q-1)^{l-1}
$$

as $q>2$. Hence $B$ is a large subgroup in that case.

Let $G \cong{ }^{3} D_{4}\left(q^{3}\right)$. Then $d=1, N=12$ and $\eta_{i}=\alpha^{i-1}$ with $\alpha \neq 1$ a third root of unity, for $1 \leq i \leq 3$. Hence $|B|=q^{12}(q-1)(q-\alpha)\left(q-\alpha^{2}\right)=q^{12}\left(q^{3}-1\right)$ and

$$
|G: B|=\left(q^{8}+q^{4}+1\right)\left(q^{3}+1\right)\left(q^{2}-1\right)<2 q^{13}<q^{12}\left(q^{3}-1\right),
$$

and the assertion holds for every $q$ (including $q=2$ ).

Let $G \cong{ }^{2} E_{6}\left(q^{2}\right)$. Then $d=(3, q+1), N=36, \eta_{1}=\cdots=\eta_{4}=1, \eta_{5}=\eta_{6}=-1$,

$$
|B|=\frac{1}{d} q^{36}(q-1)^{4}(q+1)^{2}
$$

and

$$
|G: B|=\left(q^{12}-1\right)\left(q^{9}+1\right)\left(q^{8}-1\right)\left(q^{6}-1\right)\left(q^{5}+1\right)\left(q^{2}-1\right) /(q-1)^{4}(q+1)^{2} .
$$

Here

$|G: B|<2 q^{11} q^{8} 2 q^{7} 2 q^{5}\left(q^{5}+1\right)=2^{3} q^{31}\left(q^{5}+1 \quad\right.$ and $\quad q^{5}(q-1)^{4}(q+1)>2^{3}\left(q^{5}+1\right)$,

which shows the assertion.

Let $G \cong{ }^{2} F_{4}\left(q^{2}\right)$. Then $d=1, N=24, \eta_{1}=\eta_{2}=1$ and $\eta_{3}=\eta_{4}=-1$. So

$$
|B|=q^{24}(q-1)^{2}(q+1)^{2}=q^{24}\left(q^{2}-1\right)^{2}
$$

and

$$
|G: B|=\left(q^{12}+1\right)\left(q^{8}-1\right)\left(q^{6}+1\right)\left(q^{2}-1\right) /\left(q^{2}-1\right)^{2} .
$$

Now let $r:=q^{2}=2^{2 m+1}>2$. Then

$$
|G: B|=\left(r^{6}+1\right)\left(r^{3}+r^{2}+r+1\right)\left(r^{3}+1\right) \leq\left(r^{6}+1\right) 2 r^{3}\left(r^{3}+1\right)<r^{12}(r-1)^{2}=|B|
$$

and $B$ is a large subgroup of $G$.

Let $G \cong{ }^{2} G_{2}\left(q^{2}\right)$. Then $d=1, N=6, \eta_{1}=1$ and $\eta_{2}=-1$. Then

$$
|B|=q^{6}\left(q^{2}-1\right) \quad \text { and } \quad|G: B|=\left(q^{6}+1\right)
$$

and the assertion holds in all cases.

We note that Proposition 2.2 cannot be extended to the case $q=2$, but a Borel subgroup is a large subgroup of $G$ if $G \cong{ }^{3} D_{4}(2)$ (as shown in the proof of Proposition 2.2).

Next we consider the linear groups defined over GF(2). We have the following general result. 
Proposition 2.3. Let $G$ be a special linear group of rank $l \geq 2$ defined over the field with $q$ elements. Let $V$ be the natural module for $T$ and $\left(V_{1}, V_{l}\right)$ be two subspaces of dimension one and $l$, respectively, such that $V_{1} \subseteq V_{l}$. Let $P_{i}$ be the stabiliser of $V_{i}$ in $T$, for $i=1$, l. If $(l, q) \neq(2,2)$, then $R:=P_{1} \cap P_{l}$ is a large subgroup of $G$, and $\operatorname{Con}_{G}(R)=\operatorname{Con}_{\operatorname{Aut}(G)}(R)$.

Proof. Recall that the field and diagonal automorphisms of $G$ act on the set of maximal parabolic subgroups of type $i$, for $1 \leq i \leq l$ [1] and that the graph automorphisms interchange the sets of maximal parabolics of type 1 and $l$. Since $P_{l}$ acts transitively on the one-dimensional subspaces of $V_{l}$, it follows that $\operatorname{Con}_{G}(R)=\operatorname{Con}_{\operatorname{Aut}(G)}(R)$.

Then $n:=|G: R|$ is the number of flags $\left(W_{1}, W_{l}\right)$, where $W_{i}$ an $i$-dimensional subspace of $V$ and $W_{1} \subseteq W_{l}$. We have $n=\left(q^{l+1}-1\right)\left(q^{l}-1\right) /(q-1)^{2}$. As

$$
|G|=\frac{1}{d} q^{l(l+1) / 2}\left(q^{l+1}-1\right) \cdots\left(q^{2}-1\right),
$$

where $d=(q-1, l+1)$, we get $|R|=(1 / d) q^{l(l+1) / 2}\left(q^{l-1}-1\right) \cdots\left(q^{2}-1\right)(q-1)^{2}$.

We have to show that $|G: R| \leq|R|$. If $l=2$ and $q \geq 3$ then

$$
|G: R|=\frac{\left(q^{3}-1\right)\left(q^{2}-1\right)}{(q-1)^{2}}=\left(q^{2}+q+1\right)(q+1)<\frac{1}{q-1} q^{3}(q-1)^{2} \leq|R| .
$$

If $l=3$ then

$$
|G: R|=\frac{\left(q^{4}-1\right)\left(q^{3}-1\right)}{(q-1)^{2}}<\frac{1}{q-1} q^{6}\left(q^{2}-1\right)(q-1)^{2} \leq|R|,
$$

and if $l \geq 4$ then

$$
|G: R|=\frac{\left(q^{l+1}-1\right)\left(q^{l}-1\right)}{(q-1)^{2}}<q^{2 l+1}<q^{l(l+1) / 2}<|R|,
$$

completing the proof.

Notice that the assertion of Proposition 2.3 is false for $G \cong L_{3}(2)$.

Corollary 2.4. Let $G$ be a simple group of Lie type. Then $G$ has a proper large subgroup $H$ such that $\operatorname{Con}_{G}(H)=\operatorname{Con}_{\operatorname{Aut}(G)}(H)$.

Proof. If $T \cong{ }^{3} D_{4}(2)$ or if $G$ is not defined over $G F(2)$, then the assertion follows by Proposition 2.2 and the remark after it. Therefore we may assume that $G$ is defined over $\mathrm{GF}(2)$.

If $G$ is of type $A_{l}, l>2$, then the statement is a consequence of Proposition 2.3. If

$$
G \cong B_{2}(2)^{\prime} \cong A_{6} \cong L_{2}(9), \quad G \cong A_{2}(2) \cong L_{3}(2) \cong L_{2}(7) \text { or } \quad G \cong G_{2}(2)^{\prime} \cong U_{3}(3),
$$

then we obtain the assertion by Proposition 2.2. If $G$ is as listed in Table 2, then $H$ is a large subgroup of $G$ such that $\operatorname{Con}_{G}(H)=\operatorname{Con}_{\text {Aut }(G)}(H)$ (the details are taken from [2]). 
TABLE 2. Large subgroups $H$ such that $\operatorname{Con}_{G}(H)=\operatorname{Con}_{\mathrm{Aut}(G)}(H)$.

\begin{tabular}{llrr}
\hline $\mathrm{G}$ & $\mathrm{H}$ & $|H|$ & $|G: H|$ \\
\hline$D_{4}(2)$ & $3^{4}: 2^{3} \cdot S_{4}$ & 15552 & 11200 \\
$F_{4}(2)$ & {$\left[2^{20}\right] A_{6} \cdot 2$} & 754974720 & 4385745 \\
${ }^{2} F_{4}\left(2^{2}\right)^{\prime}$ & $2 \cdot\left[2^{8}\right]: 5: 4$ & 10240 & 1755 \\
\hline
\end{tabular}

If $G$ is one of the remaining groups of Lie type with $q=2$, that is, $G$ is isomorphic to one of the groups

$$
B_{l}(2), D_{l}(2)(l \geq 5), E_{6}(2), E_{7}(2), E_{8}(2),{ }^{2} A_{l}\left(2^{2}\right),{ }^{2} D_{l}\left(2^{2}\right),{ }^{2} E_{6}\left(2^{2}\right),
$$

then it is easily verified that the large subgroup $H$ of $G$ given by [3, Table II] satisfies $\operatorname{Con}_{G}(H)=\operatorname{Con}_{\mathrm{Aut}(G)}(H)$. This completes the proof.

It remains to consider the alternating groups.

Proposition 2.5. Let $G \cong A_{n}, n \geq 5$. Then $G$ has a proper large subgroup $H$ such that $\operatorname{Con}_{G}(H)=\operatorname{Con}_{\operatorname{Aut}(G)}(H)$.

Proof. The case $G=A_{6} \cong L_{2}(9)$ has already been handled in Proposition 2.2. Thus we may assume that $n \neq 6$, in which case $\operatorname{Aut}(G)=S_{n}$. Let $H$ be a point stabiliser in $G=A_{n}$; then $\operatorname{Con}_{A_{n}}(H)=\operatorname{Con}_{S_{n}}(H)$, and clearly $H$ is large in $G=A_{n}$. This completes the proof.

Now Proposition 1.3 follows by Proposition 2.1, Corollary 2.4 and Proposition 2.5.

The following proposition will be used in the proof of Theorem 1.4

Proposition 2.6. Let $G$ be a simple nonabelian group. Then $G$ has a proper subgroup $H$ such that $|H| \geq|G: H|^{\beta}$ and $\operatorname{Con}_{G}(H)=\operatorname{Con}_{\mathrm{Aut}(G)}(H)$.

Proof. In view of Proposition 1.3, it remains to consider the case $G=O$ ' Nan. By [2] $G$ has a (maximal) subgroup $H \cong J_{1},|H|=175560,|G: H|=2624832$, such that $\operatorname{Con}_{G}(H)=\operatorname{Con}_{\text {Aut }(G)}(H)$. Since $|H|=|G: H|^{\beta}$, the proof is complete.

REMARK 2.7. The number $\beta$ cannot be replaced by a larger constant in Proposition 2.6. Indeed, let $T:=O$ 'Nan and let $A<T$ be such that $\operatorname{Con}_{T}(A)=\operatorname{Con}_{\text {Aut }(T)}(A)$. We show that $|A| \leq|T: A|^{\beta}$. Set $G=\operatorname{Aut}(T)$. By Frattini's argument $G=T N_{G}(A)$, and so

$$
|T: A| \geq\left|T: T \cap N_{G}(A)\right|=\left|G: N_{G}(A)\right| .
$$

The list of maximal subgroups of $G=\operatorname{Aut}(T) \cong O^{\prime} N a n: 2$ is determined in [7]. By this list $S:=J_{1} \times 2$ is the largest maximal subgroup of $\operatorname{Aut}(T)$ distinct from $T$. Thus $|T: A| \geq|G: S|=2624832$, which implies that $|A| \leq|T: A|^{\beta}$ as required.

As noted in Section 1, the following shows that Theorem 1.4 cannot be improved by replacing $\beta$ by a larger constant. 
Proposition 2.8. Let $T=O$ 'Nan and $G=\operatorname{Aut}(T)$. Then $|\operatorname{Sec}(M)| \leq|G: M|^{\beta}$ for each maximal subgroup $M$ of $G$.

Proof. Let $M$ be a maximal subgroup of $G$. If $M=T$ then $\operatorname{Sec}(M)=1$, so we may assume that $G=M T$ and $M \cap T<T$. For $g \in G$ there exist $u \in M, t \in T$ such that $g=u t$ and so $(M \cap T)^{g}=M^{g} \cap T=M^{t} \cap T=(M \cap T)^{t}$. This shows that $\operatorname{Con}_{T}(M \cap T)=\operatorname{Con}_{G}(M \cap T)$, and thus by Remark 2.7,

$$
|M \cap T| \leq|T: M \cap T|^{\beta}=|M T: M|^{\beta}=|G: M|^{\beta} .
$$

Since $T / 1$ is a chief factor of $G$ and $T \not \leq M, 1<M$, we have $\operatorname{Sec}(M)=M \cap T$, so by the above $|\operatorname{Sec}(M)| \leq|G: M|^{\beta}$ as required.

\section{Proofs of Theorems 1.2, 1.4, 1.5 and 1.6}

We start with the proof of our classification-free result.

Proof of Theorem 1.5. Suppose that (2) does not hold. Then there exist a nonabelian chief factor $K / L$ of $G$, and a large proper subgroup $B / L$ of $K / L$ such that $\operatorname{Con}_{K}(B)=$ $\operatorname{Con}_{G}(B)$. We shall show that $G / L$ has a maximal subgroup $M / L$ such that $|\operatorname{Sec}(M)| \geq$ $|G: M|$, so (1) fails. There is no loss of generality here in assuming that $L=1$. By Frattini's argument, $G=K N_{G}(B)$. Since $B$ is not normal in $G$ we can choose $M$, a maximal subgroup of $G$ containing $N_{G}(B)$. Then $M \nsupseteq K$ and $K$ is minimal normal, so $\operatorname{Sec}(M)=M \cap K$. But $M \cap K \geq B$ and $B$ is a large subgroup of $K$. Thus $|M \cap K| \geq$ $|K: M \cap K|=|G: M|$, which implies that $|\operatorname{Sec}(M)| \geq|G: M|$.

Conversely, suppose that (1) does not hold and let $M$ be a maximal subgroup of $G$ with $|\operatorname{Sec}(M)| \geq|G: M|$. Let $K / L$ be a chief factor of $G$ satisfying $L \leq M$ and $K \not \leq M$. Then $G=K M$, implying that $|G: M|=|K: M \cap K|$ and so $|(M \cap K) / L| \geq|K: M \cap K|$. Thus $(M \cap K) / L$ is a large proper subgroup of $K / L$. (Notice that $K / L$ is nonabelian, since otherwise $M \cap K / L \triangleleft G / L$, contradicting the fact that $K / L$ is a chief factor.) In order to see that (2) fails, it is left to show that $\operatorname{Con}_{K}(M \cap K)=\operatorname{Con}_{G}(M \cap K)$. Let $g \in G$; then $g=m k$, where $m \in M, k \in K$. Thus $(M \cap K)^{g}=(M \cap K)^{k}$, and the proof is completed.

We proceed with a useful lemma.

Lemma 3.1. Let $G$ be a group, $N \unlhd G, N=T^{m}$, where $T$ is a simple nonabelian group. Suppose that $B \leq T$ and $\operatorname{Con}_{T}(B)=\operatorname{Con}_{\mathrm{Aut}(T)}(B)$. Let $A:=B^{m}$ be a subgroup of $N$. Then $\operatorname{Con}_{N}(A)=\operatorname{Con}_{G}(A)$.

Proof. By construction

$$
\operatorname{Aut}(N)=\operatorname{Aut}(T) w r S_{m}=N_{\operatorname{Aut}(N)}(A) \operatorname{Aut}(T)^{m} .
$$

Since each $g \in G$ acts on $N$ (by conjugation) like an element of $\operatorname{Aut}(N)$, the assertion now follows from the assumption that $\operatorname{Con}_{T}(B)=\operatorname{Con}_{\operatorname{Aut}(T)}(B)$. 
Theorems 1.2, 1.4 and 1.6 can now be proved.

Proof of Theorem 1.2. Let $G$ be a group such that $|\operatorname{Sec}(M)|<|G: M|$ for all maximal subgroups $M$ of $G$. Suppose to the contrary that $G$ has a chief factor $K / L=T^{m}, T$ is a simple nonabelian group and $T \nRightarrow O^{\prime} N a n$. By Proposition 1.3 there exists a proper large subgroup $B$ of $T$ such that $\operatorname{Con}_{T}(B)=\operatorname{Con}_{\text {Aut }(T)}(B)$. Let $A=B^{m}$, a subgroup of $K / L$. Then it is easily verified that $A$ is a proper large subgroup of $K / L$, and by Lemma 3.1, $\operatorname{Con}_{K / L}(A)=\operatorname{Con}_{G / L}(A)$. Let $H$ be the preimage of $A$ in $G$; then clearly $\operatorname{Con}_{K}(H)=\operatorname{Con}_{G}(H)$, so condition (2) of Theorem 1.5 is not satisfied by $G$. Since condition (1) of the same theorem is satisfied, we reach the desired contradiction.

Proof of Theorem 1.4. The only if part is known, as mentioned in Section 1. We prove the if part. Let $G$ be a minimal counterexample. Since the condition on the $c$-sections of $G$ is inherited by quotients of $G, G / N$ is solvable for each $1<N \unlhd G$. Hence $G$ has a unique minimal normal subgroup $N$, and $N=T^{m}$, where $T$ is a simple nonabelian group. Furthermore,

$$
N=T^{m} \leq G \leq \operatorname{Aut}(T) w r S_{m}=\operatorname{Aut}(N) .
$$

By Proposition 2.6 there exists a proper subgroup $H$ of $T$ such that $|H| \geq|G: H|^{\beta}$ and $\operatorname{Con}_{T}(H)=\operatorname{Con}_{\text {Aut }(T)}(H)$. Define $A=H^{m}$, a subgroup of $N$. Then it is easily verified that $|A| \geq|N: A|^{\beta}$, and by Lemma 3.1, $\operatorname{Con}_{N}(A)=\operatorname{Con}_{G}(A)$. Frattini's argument leads to $G=N N_{G}(A)$. Notice that $A<N$ forces that $A$ is not normal in $G$. Let $M$ be a maximal subgroup of $G$ containing $N_{G}(A)$. Then $N \not \leq M$ and, since $N$ is minimal normal, $\operatorname{Sec}(M) \cong M \cap N$. Now $M \cap N \geq A$, implying that $|M \cap N| \geq|N: A|^{\beta} \geq \mid N$ : $\left.M \cap N\right|^{\beta}$. But since $G=M N$ we have $|N: M \cap N|=|G: M|$. Hence $|\operatorname{Sec}(M)| \geq \mid G$ : $\left.M\right|^{\beta}$, the desired contradiction.

Proof of Theorem 1.6. Assume that the theorem is false and let $G$ be a minimal counterexample. Suppose first that $G$ does not have proper nontrivial characteristic subgroups. Then, since $G$ is not a $p$-group, $G=T^{m}$, where $T$ is a simple nonabelian group. Moreover, by assumption $T \nsubseteq O^{\prime} N a n$. By Proposition 1.3 there exists $S<T$ such that $\operatorname{Con}_{T}(S)=\operatorname{Con}_{\operatorname{Aut}(T)}(S)$ and $S$ is large in $T$. Set $H=S^{m}$, a subgroup of $G=T^{m}$. Then $H$ is a proper large subgroup of $G$, and $\operatorname{Con}_{G}(H)=\operatorname{Con}_{\operatorname{Aut}(G)}(H)$ by Lemma 3.1. Therefore, $G$ is not a counterexample in this case. Hence we may assume from now on that $G$ has proper nontrivial characteristic subgroups.

Let $K$ be a minimal characteristic subgroup of $G$. Then $K=T^{m}$, where $T$ is a simple group ( $T$ may be of prime order). Suppose that $|G / K|$ is divisible by at least two primes. Then, since $G / K$ is not a counterexample, there exists $K<H<G$ such that $H / K$ is large in $G / K$ and $\operatorname{Con}_{G / K}(H / K)=\operatorname{Con}_{\operatorname{Aut}(G / K)}(H / K)$. Let $\alpha \in \operatorname{Aut}(G)$; then $\alpha$ induces an automorphism $\bar{\alpha}$ of $G / K$ and $(H / K)^{\bar{\alpha}}=H^{g} / K$ for some $g \in G$. Thus $H^{\alpha}=H^{g}$, and it follows that $\operatorname{Con}_{G}(H)=\operatorname{Con}_{\operatorname{Aut}(G)}(H)$. Since $H$ is large in $G$, we deduce that $G$ is not a counterexample.

It remains, therefore, to consider the case where $G / K$ is a nontrivial $p$-group for a prime $p$. If $K$ is elementary abelian then $K$ is a $q$-group for a prime $q$ distinct from $p$. 
Now, either a Sylow $p$-subgroup or a Sylow $q$-subgroup of $G$ is large in $G$. Since this Sylow subgroup, say $H$, satisfies $\operatorname{Con}_{G}(H)=\operatorname{Con}_{\operatorname{Aut}(G)}(H)$ by Sylow's theorem, we deduce again that $G$ is not a counterexample. Finally, suppose that $K$ is nonsolvable. Let $R$ be a nontrivial Sylow subgroup of $K$. By Frattini's argument, $G=K N_{G}(R)$ and so either $K$ or $N_{G}(R)$ is a proper large subgroup of $G$. Denote this large subgroup by $H$. If $H=K$ then $\operatorname{Con}_{G}(H)=\operatorname{Con}_{\text {Aut }(G)}(H)=\{H\}$. If $H=N_{G}(R)$, notice that for $\alpha \in \operatorname{Aut}(G)$ there exists $u \in K$ such that $R^{\alpha}=R^{u}$. Thus $H^{\alpha}=H^{u}$, and it follows that $\operatorname{Con}_{G}(H)=\operatorname{Con}_{\operatorname{Aut}(G)}(H)$. This shows that $G$ is not a counterexample in this case, as well. The proof is now completed.

\section{References}

[1] R. Carter, Simple Groups of Lie Type (Wiley, London, 1972).

[2] J. H. Conway, R. T. Curtis, S. P. Norton, R. A. Parker and R. A. Wilson, Atlas of Finite Groups (Clarendon, Oxford, 1985).

[3] A. Lev, 'On large subgroups of finite groups', J. Algebra 152 (1992), 434-438.

[4] S. Li and W. Shi, 'A note on the solvability of groups', arXiv:math/0509377v2 (2005).

[5] L. Shirong and Y. Wang, 'On $c$-section and $c$-index of finite groups', J. Pure Appl. Algebra 151 (2000), 300-319.

[6] Y. Wang, 'C-normality of groups and its properties', J. Algebra 180 (1998), 954-965.

[7] R. A. Wilson, 'The maximal subgroups of the O'Nan group', J. Algebra 97 (1985), 467-473.

\section{BARBARA BAUMEISTER, Fakultät für Mathematik, Universität Bielefeld, Postfach 1001 31, D-33501 Bielefeld, Germany \\ e-mail: baumeist@math.uni-bielefeld.de}

GIL KAPLAN, School of Computer Sciences, The Academic College of Tel-Aviv-Yaffo, 2 Rabenu Yeruham st., Tel-Aviv 64044, Israel 